\title{
3D Continuous and Discrete Modeling of Bifurcations in Geomaterials
}

\author{
Florent PRUNIER, Félix DARVE, Luc SIBILLE and François NICOT
}

\subsection{Introduction}

Geomaterials are strongly non-associated materials (dilatancy angle is generally lower than friction angle by $25^{\circ}$ as a very rough estimate, while both angles should be close for associated materials). Due to this, the elastoplastic tensor is not symmetric and various types of bifurcations can be expected before reaching MohrCoulomb plastic limit condition. In fact, two examples of such bifurcations are well recognized. On the one hand, experiments have shown that plastic strain localization can appear before the stress peak in drained triaxial compressions [DER 04]. We have here a bifurcation of the strain mode from a quasi-homogenous mode into a localized one. On the other hand, the deviatoric stress peak of loose sand subjected to undrained triaxial compressions (such a peak is inside Mohr-Coulomb's surface) gives rise to a diffuse mode of failure if a small axial force is added [KHO 06]. The existence of such bifurcations before the Mohr-Coulomb plastic limit condition is established for all non-associated materials after the elastoplastic theory. As nonassociativeness is generally linked to mean pressure dependent plastic behavior, such features can be expected for other mean pressure dependent yield conditions such as the Drucker-Prager criterion.

To investigate these bifurcations, various criteria are available, such as vanishing of the determinant of the elastoplastic matrix, or of the acoustic matrix, or of the symmetric part of the elastoplastic matrix, etc. If we except flutter instabilities, one 
of these criteria plays a particular role because it constitutes a lower bound for all these criteria [BIG 90]. This is the so-called "second-order work criterion", which is equivalent to the vanishing of the determinant of the symmetric part of the elastoplastic matrix for incrementally piece-wise linear elastoplastic constitutive relations [DAR 04].

The second-order work criterion has been extensively studied in axisymmetric conditions [DAR 04] and in plane strain conditions [NIC 07b]. The equation of the boundary of the bifurcation domain has been established and the directional character of this criterion has led to the existence of cones of unstable stress directions.

The first purpose of this chapter is to generalize these results to 3D conditions in fixed principal stress-strain axes. By considering an incrementally piece-wise linear constitutive relation the analytical equations of the boundary of the bifurcation domain and of the instability cones are established explicitly, then plotted in 3D principal stress space.

Even if some partial experimental validations of these results are now available [CHU 03], these experiments are delicate to perform essentially because bifurcations are very sensitive to any imperfection or perturbation. Thus it is interesting to try to simulate these material instabilities using a numerical model of granular materials. These models in the line of molecular dynamic methods are now available [MAG 98] and it is possible to simulate the behavior of cubical specimens of granular materials [SIB 07a].

The second part of this chapter is thus devoted to a direct numerical investigation of second-order work criterion. First by checking the sign of secondorder work at various stress-strain states and for various stress directions, a bifurcation domain and instability cones are exhibited. Then, by considering the precise theoretical conditions for an effective failure:

- a stress-strain state inside the bifurcation domain;

- an incremental stress direction inside the instability cone;

- proper loading parameters allowing the failure to develop;

some failures have been obtained numerically, which are characterized by exponentially growing strains, bursts of kinetic energy and diffuse failure modes. 


\subsection{D bifurcations exhibited by an incrementally non-linear constitutive relation}

This first part is devoted to the study of the second-order work criterion using incrementally piecewise constitutive relation in an analytical and numerical framework. Numerical results will also be presented with an incrementally nonlinear relation. This criterion [HIL 58] specifies that a mechanical specimen is stable if:

$$
\forall(\underline{\underline{d \sigma}}: \underline{\underline{d \varepsilon}}) \text { linked by their constitutive relation } d^{2} W=\underline{\underline{d \sigma}}: \underline{\underline{d \varepsilon}}>0
$$

$\underline{\underline{d \sigma}}$ denotes the Cauchy stress increment tensor, and $\underline{\underline{d \varepsilon}}$ the small strain increment tensor. The converse is false, thus if $d^{2} W \leq 0$, the body is in a potentially unstable state. We will show further that the occurrence of instability depends on controlled loading parameters and direction of loading. In a first section, a brief reminder of the incrementally non-linear and octo-linear constitutive models of Darve will be given [DAR 95]. Then in a second section, the 3D limit of the bifurcation domain and 3D "cones" of unstable loading directions will be displayed for these two models. We emphasize that all analytical results in this part are valid for every incrementally piece-wise linear elastoplastic model. Only numerical results displayed in figures are restricted to the last two models.

\subsubsection{Incrementally non-linear and piecewise linear relations}

For simplification purposes, symmetric second-order tensors $\underline{\underline{d \sigma}}$ and $\underline{\underline{d \varepsilon}}$ will henceforth be represented by a column vector of dimension 6 and denoted: $\underline{d \sigma}$ and $\underline{d \varepsilon}$. Concerning these constitutive relations, it is worth noting that they are not related to classic concepts of elastoplasticity and therefore assumptions of:

- strain decomposition into an elastic and a plastic part;

- existence of an elastic limit;

- existence of a flow rule.

To describe the non-linear behavior of geomaterials, an incrementally non-linear relation of second-order is used and written in principal axes as follows: 


$$
\left\{\begin{array}{l}
d \varepsilon_{1} \\
d \varepsilon_{2} \\
d \varepsilon_{3}
\end{array}\right\}=\frac{1}{2}\left[\underline{\underline{N}}^{+}+\underline{\underline{N}}^{-}\right]\left\{\begin{array}{c}
d \sigma_{1} \\
d \sigma_{2} \\
d \sigma_{3}
\end{array}\right\}+\frac{1}{2\|\underline{\underline{d \sigma}}\|}\left[\underline{\underline{N}}^{+}+\underline{\underline{N}}^{-}\right]\left\{\begin{array}{c}
d \sigma_{1}^{2} \\
d \sigma_{2}^{2} \\
d \sigma_{3}^{2}
\end{array}\right\}
$$

with:

$$
\underline{N}^{ \pm}=\left[\begin{array}{ccc}
\frac{1}{E_{1}^{ \pm}} & -\frac{v_{21}^{ \pm}}{E_{2}^{ \pm}} & -\frac{v_{31}^{ \pm}}{E_{3}^{ \pm}} \\
-\frac{v_{12}^{ \pm}}{E_{1}^{ \pm}} & \frac{1}{E_{2}^{ \pm}} & -\frac{v_{32}^{ \pm}}{E_{3}^{ \pm}} \\
-\frac{v_{13}^{ \pm}}{E_{1}^{ \pm}} & -\frac{v_{23}^{ \pm}}{E_{2}^{ \pm}} & \frac{1}{E_{3}^{ \pm}}
\end{array}\right]
$$

Coefficients $E_{i}^{+}$and $v_{i j}^{+}$are defined on generalized triaxial loading paths when $d \sigma_{i}>0$ and respectively $E_{i}^{-}$and $v_{i j}^{-}$when $d \sigma_{i}<0$. For $d \sigma_{i}=0$, it can be verified that the relation is continuous [GUD 79]; see [DAR 95] to find out more about this constitutive relation and particularly how the tangent moduli and Poisson's ratios are evolving with the stress-strain history. At one dimension, this relation is piecewise linear. By extrapolation F. Darve defined the octo-linear model (eight tensorial zones) ${ }^{1}$ as follows with the previous notations:

$$
\left\{\begin{array}{l}
d \varepsilon_{1} \\
d \varepsilon_{2} \\
d \varepsilon_{3}
\end{array}\right\}=\frac{1}{2}\left[\underline{\underline{N}}^{+}+\underline{\underline{N}}^{-}\right]\left\{\begin{array}{c}
d \sigma_{1} \\
d \sigma_{2} \\
d \sigma_{3}
\end{array}\right\}+\frac{1}{2}\left[\underline{\underline{N}}^{+}+\underline{\underline{N}}^{-}\right]\left\{\begin{array}{l}
\left|d \sigma_{1}\right| \\
\left|d \sigma_{2}\right| \\
\left|d \sigma_{3}\right|
\end{array}\right\}
$$

In this expression, the eight tensorial zones are explicit, and relationship [9.4] is identical to the following eight linear relations:

1 A tensorial zone is a domain of the loading space in which the incremental constitutive relation is linear. A classical elastoplastic model has two tensorial zones: one for loading and the second for unloading. 


$$
\underline{d \varepsilon}=\left(\underline{\underline{N_{i}}}\right)_{i=1 \ldots 8} \underline{\frac{d \sigma}{1}}
$$

with $\left(\underline{\underline{N_{i}}}\right)_{i=1 \ldots 8}$ the 8 matrices $\underline{N}$ where indices $(+)$ are affected to the column $d \sigma_{j}$ if $d \sigma_{j}>0$, and (-) if $d \sigma_{j}<0 j \in\{1 ; 2 ; 3\}$. In the next sections, relation [9.5] will be used for analytical calculations. In fact, calculations are assumed to be performed in a given tensorial zone.

\subsubsection{D analysis of the second-order work with phenomenological constitutive models}

As already shown in the past years [DAR 00], the second-order work depends essentially on the loading direction. After a given loading path, the material has reached some stress-strain states which could be potentially unstable, even inside the plastic limit condition. From this strain-stress state situated inside the plastic limit condition, some loading directions can lead to the failure and the others not. If no loading direction leads to the failure, the mechanical state is considered stable, otherwise potentially unstable. In the global 3D loading space, the limit where the material is potentially unstable or stable is called the limit of the bifurcation domain. [DAR 04] and [KHO 06] have determined this limit of bifurcation and the cones of unstable loading directions for axisymmetric and plane strain conditions respectively. We intend to make a generalization in $3 \mathrm{D}$ conditions here.

To make the link between the vanishing of the second-order work and the existence of a peak in a plane of conjugated loading variables, we choose to define strain proportional loading paths as in equation [9.6]. These strain paths allow the scanning of all loading directions of the 3D strain space.

$$
\left\{\begin{array}{c}
d \varepsilon_{1}=c s t \quad c s t \in \Re \\
d \varepsilon_{1}+R d \varepsilon_{3}=0 \quad R \in \Re^{*} \\
d \varepsilon_{2}-R^{\prime} d \varepsilon_{3}=0 \quad R^{\prime} \in \Re
\end{array}\right.
$$

$\mathfrak{R}$ denotes the set of all real numbers, while $\mathfrak{R}^{*}$ is the set of real numbers except the element 0 . Figure 9.1 shows the results for these strain paths in invariant planes. 

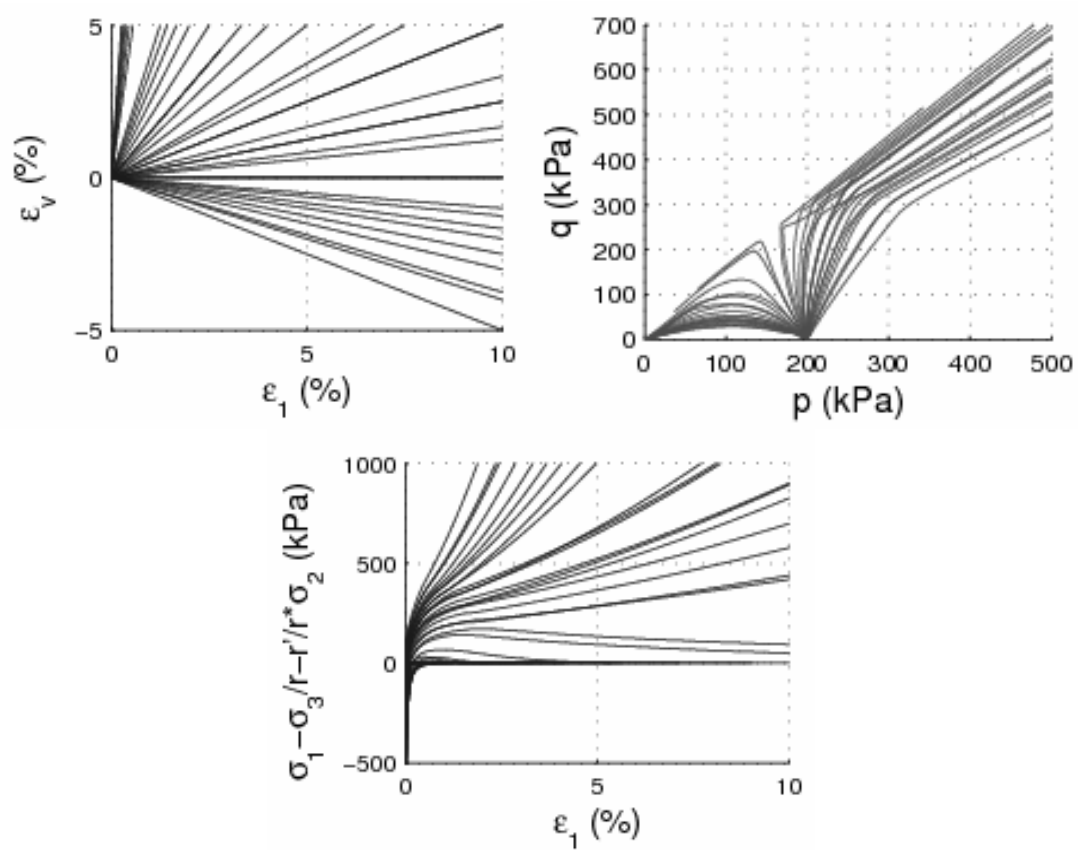

$$
p=\frac{1}{3} \operatorname{tr}(\underline{\underline{\sigma}}), q=\sqrt{\frac{3}{2}}\|\operatorname{dev}(\underline{\underline{\sigma}})\|, \operatorname{dev}(\underline{\underline{\sigma}})=\underline{\underline{\sigma}}-p \underline{\underline{I}}
$$

Figure 9.1. Response for proportional strain loading paths obtained with the octo-linear model. $R \in[0.1 ; 2]$ and, $R^{\prime} \in[0 ; 1]$

Knowing that:

$$
\begin{gathered}
\left(d \sigma_{1}-\frac{d \sigma_{3}}{R}-\frac{R^{\prime}}{R} d \sigma_{2}\right) d \varepsilon_{1}+\left(d \varepsilon_{1}+R d \varepsilon_{3}\right)\left(\frac{d \sigma_{3}}{R}+\frac{R^{\prime}}{R} d \sigma_{2}\right)+\left(d \varepsilon_{2}-R^{\prime} d \varepsilon_{3}\right) d \sigma_{2} \\
=d \sigma_{1} d \varepsilon_{1}+d \sigma_{2} d \varepsilon_{2}+d \sigma_{3} d \varepsilon_{3}=d^{2} W
\end{gathered}
$$

An admissible set of conjugated variables for the second-order work, considering loading paths of [9.6], can be the following: 


$$
\left[\begin{array}{c}
d \sigma_{1}-\frac{d \sigma_{3}}{R}-\frac{R^{\prime}}{R} d \sigma_{2} \\
d \varepsilon_{1}+R d \varepsilon_{3} \\
d \varepsilon_{2}-R^{\prime} d \varepsilon_{3}
\end{array}\right]=\underline{=}\left[\begin{array}{c}
d \varepsilon_{1} \\
\frac{d \sigma_{3}}{R}+\frac{R^{\prime}}{R} d \sigma_{2} \\
d \sigma_{2}
\end{array}\right]
$$

Taking into account kinematics conditions from equation [9.6], the second-order work will vanish for the class of loading paths defined by: $d \varepsilon_{1}+R d \varepsilon_{3}=0$ and $d \varepsilon_{2}-R^{\prime} d \varepsilon_{3}=0$ at the peak of $d \sigma_{1}-\frac{d \sigma_{3}}{R}-\frac{R^{\prime}}{R} d \sigma_{2}$ versus $d \varepsilon_{1}$ in Figure 9.1. This state also corresponds to the vanishing value of the determinant of $\underline{\underline{S}}$. If we locate these peaks in the 3D stress space, it can be shown that they are situated strictly inside the plastic limit condition. Thus, we found a class of loading paths where instability occurs strictly inside the plasticity criterion, and this instability corresponds to the vanishing value of the second-order work.

If we define the matrix $\underline{\underline{M}}$ so that:

$$
\underline{d \sigma}=\underline{\underline{M}} \underline{d \varepsilon}
$$

it can be verified that:

$$
\operatorname{det}(\underline{\underline{S}})=0 \Leftrightarrow \underline{\underline{d \varepsilon}} \underline{\underline{\underline{S}}} \underline{\underline{d \varepsilon}}=0
$$

with $M_{S}$ the symmetric part of $\underline{\underline{M}}$. In fact, as already written above:

$$
\operatorname{det}(\underline{\underline{S}})=0 \Leftrightarrow d^{2} W=0 \Leftrightarrow \underline{\underline{d \varepsilon}} \underline{\underline{M}} \underline{\underline{d \varepsilon}}=0 \Leftrightarrow \underline{\underline{d \varepsilon}} \underline{\underline{M_{S}}} \underline{\underline{d \varepsilon}}=0
$$

and:

$$
\underline{d \varepsilon} \underline{\underline{M_{S}}} \underline{\underline{d \varepsilon}}=0
$$

constitutes the condition on the direction of strain increments which will lead to the failure from a given stress-strain state. [9.12] is a quadric in the $3 \mathrm{D}$ incremental 
strain space with neither constant terms nor first degree terms, so [9.12] is the equation of an elliptic cone in the 3D incremental strain space. If we assume that eigenvalues of $\underline{\underline{M_{S}}}$ evolve continuously with the loading parameter and are positive at the original virgin state, there are 4 cases. First, at the beginning of the loading program, all eigenvalues are positive and [9.12] has no solution, except the center of the quadric itself, so these states are stable and out of the bifurcation limit. Then one of the eigenvalues vanishes and $\operatorname{det}\left(M_{S}\right)=0$. Thus, [9.12] admits a straight line (in the direction of the corresponding eigenvector) as a solution. From this strain-stress state, this loading direction is unstable. This state corresponds to the limit of the bifurcation domain. Continuing to increase the loading parameter, one eigenvalue is negative, and the two others are positive, then $\operatorname{det}\left(\underline{\underline{M_{S}}}\right)<0$ and [9.12] admits an elliptical cone in the 3D strain space as solution. All loading directions situated inside or on this cone lead to failure. This mechanical state is inside the bifurcation domain, but before the plastic limit. Finally, as the ultimate mathematical solution, we could obtain two eigenvalues of the opposite sign and the third one equal to zero, then $\operatorname{det}\left(\underline{\underline{M_{S}}}\right)=0$, and [9.12] admits two secant planes as a solution, but this latest case was never reached numerically by our models.

Of course dual analysis can be performed in stress space. In the same manner we also have:

$$
d^{2} W=0 \Leftrightarrow \underline{d \sigma} \underline{\underline{N}} \underline{d \sigma}=0 \Leftrightarrow \underline{\underline{d \sigma}} \underline{\underline{N_{S}}} \underline{d \sigma}=0
$$

which gives in an extended form:

$$
\frac{d \sigma_{1}^{2}}{E_{1}}+\frac{d \sigma_{2}^{2}}{E_{2}}+\frac{d \sigma_{3}^{2}}{E_{3}}-\left(\frac{v_{1}^{2}}{E_{1}}+\frac{v_{2}^{1}}{E_{2}}\right) d \sigma_{1} d \sigma_{2}-\left(\frac{v_{3}^{2}}{E_{3}}+\frac{v_{2}^{3}}{E_{2}}\right) d \sigma_{2} d \sigma_{3}-\left(\frac{v_{1}^{3}}{E_{1}}+\frac{v_{3}^{1}}{E_{3}}\right) d \sigma_{3} d \sigma_{1}=0
$$

with $\underline{\underline{N}}=\underline{\underline{M}}$ the matrix of equation [9.5], and $\underline{\underline{N_{S}}}$ the symmetric part of $\underline{\underline{N}}$. In that way we can plot the bifurcation limit and cones of unstable directions in the 3D stress space. Figure 9.2 shows the limit of the bifurcation domain for the incrementally non-linear and octo-linear relation of Darve for a dense Hostun sand. The following observations are derived from Figure 9.2: 
- the bifurcation domain of the octo-linear model is larger than that of the nonlinear model (the limit is reached before according to the loading parameter);

- there are some discontinuities with the octo-linear model due to the switching of tensorial zones;

- the limits of the bifurcation domain are noticeably conical (which corresponds to the conical structure of Mohr-Coulomb plastic limit condition), but not rigorously conical because the mechanical properties do not vary exactly in proportion with the mean stress level in the constitutive models used.
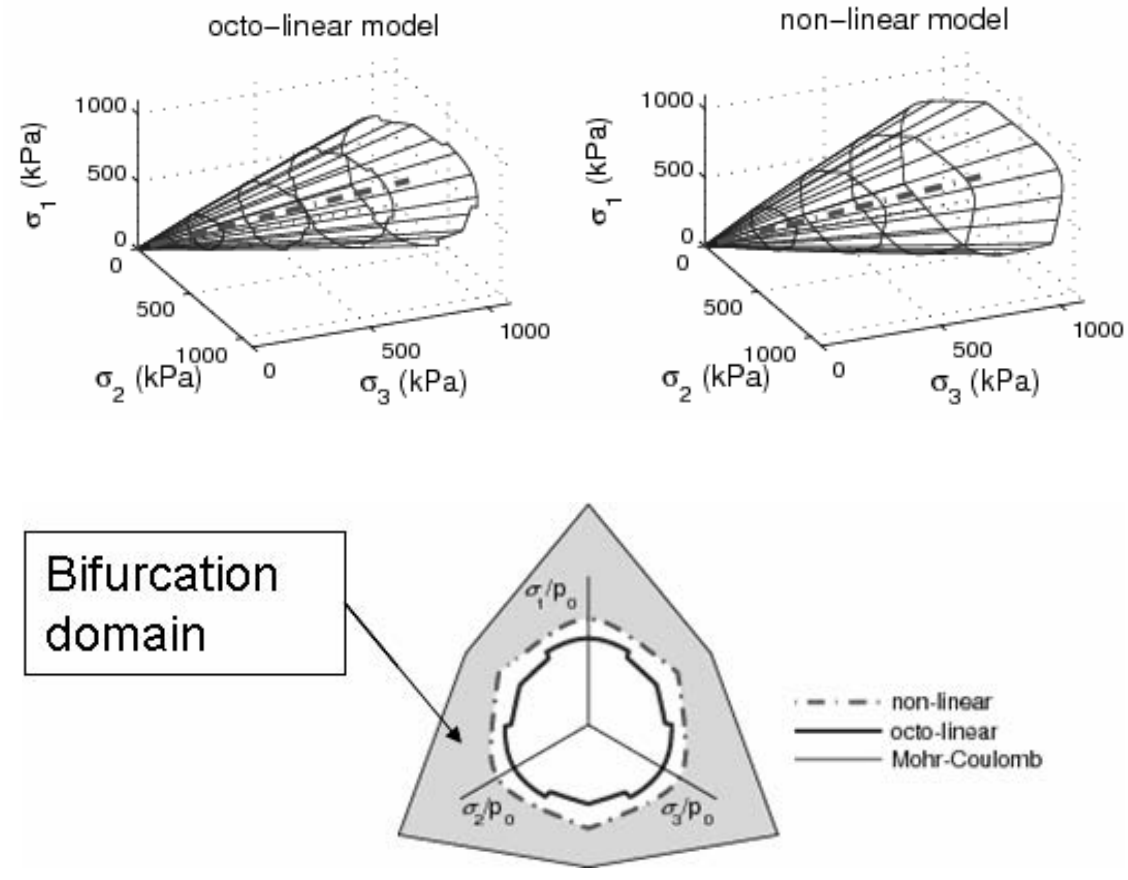

Figure 9.2. Bifurcation domain plotted with the octo- and non-linear model for a dense Hostun sand 
To plot these bifurcation domains, radial stress paths along a given direction in the deviatoric stress plane were considered until the condition $\operatorname{det}\left(\underline{\underline{N_{S}}}\right)=0$ was verified. Figure 9.3 illustrates such a stress path.

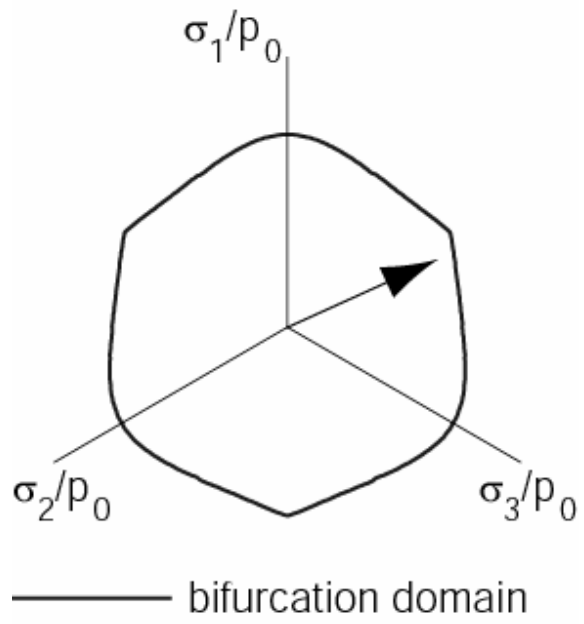

Figure 9.3. Example of stress path followed to detect the limit of the bifurcation domain

Then when we write $\operatorname{det}\left(\underline{\underline{N_{S}}}\right)=0$, we have to consider in fact $\min \left(\operatorname{det}\left(\underline{\underline{N_{S}}}\right)_{i}\right)=0$ with $\mathrm{i}=\{1, \ldots 8\}$ for the octo-linear relation, and $\mathrm{i}=\{1, \ldots, \infty\}$ for the non-linear relation. For the non-linear relation each direction of the 3D sphere of the increments of stresses can be considered as a tensorial zone. In this case, numerical results are obtained with a precision of $1^{\circ}$ on the $3 \mathrm{D}$ sphere.

We will now present and discuss cones of unstable loading directions for stressstrain states situated beyond the bifurcation limit. To do this, two procedures have been implemented. The first is purely numerical, based on Gudehus's response envelope technique [GUD 79], and proposed by [DAR 00]. The principle is as follows: a drained triaxial loading path is followed, and at a given deviatoric state, a stress increment in a 3D space direction is imposed. If the second-order work is negative or zero, the direction is thought to be unstable (numerically, all directions can be tested at each step of the triaxial path). Figure 9.4 illustrates this procedure and gives results obtained by [DAR 04] for a dense Hostun sand for axisymmetric conditions. 

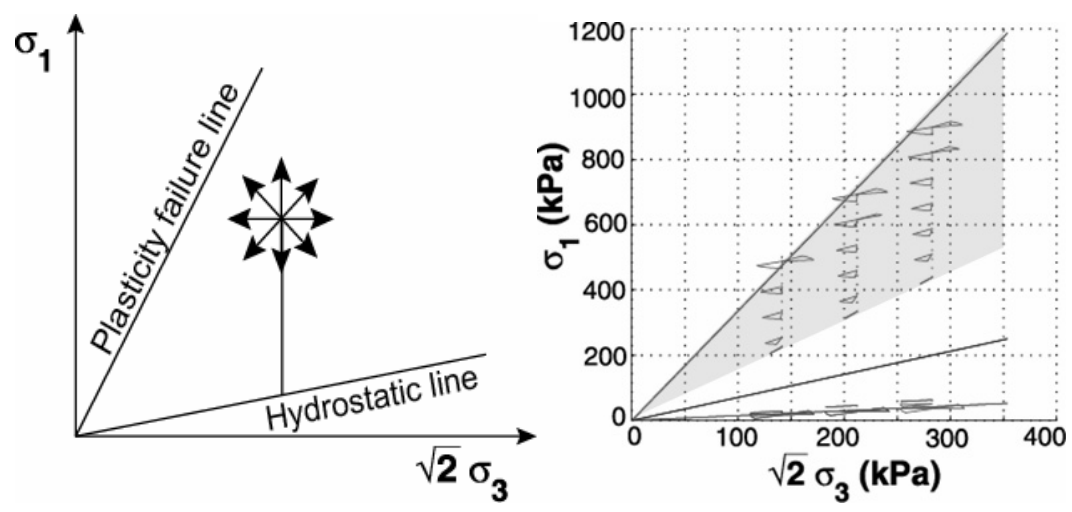

Figure 9.4. Stress probe test to find unstable cones (left) and results obtained in axisymmetric conditions (right)

The second method is simultaneously analytical and numerical. It consists of substituting current numerical values of tangent moduli and Poisson's ratios into equation [9.14]. At a given deviatoric level, there are eight equations. As a result, we have to truncate the elliptical equations so that they do not overflow their tensorial zone. This method enables us to verify that there is an agreement between the numerical and analytical results for the octo-linear model. These results are displayed in Figure 9.5. Numerical points are represented in the points cloud and analytical cones are represented by the mesh for the octo-linear model. Comparison between analytical and numerical results proves to be highly satisfying. Of course, for the non-linear model, only the first numerical method can be used because each unstable direction is an infinitesimal elliptical cone, so this structure of elliptical cone could not be preserved. In Figure 9.5 limits between each tensorial zone of the octo-linear model are represented by the three orthogonal planes. Finally, we have chosen to show four states distributed in an equidistant way between the bifurcation domain (first unstable direction) and the plasticity limit criterion, as Figure 9.6 illustrates. Considering the same deviatoric level for both models in Figure 9.5 would not have been proper for the comparison. As we have seen before in Figure 9.2, bifurcation domains are different for these two models, whereas the constitutive constants (calibrated for the same Hostun sand) are exactly the same. 

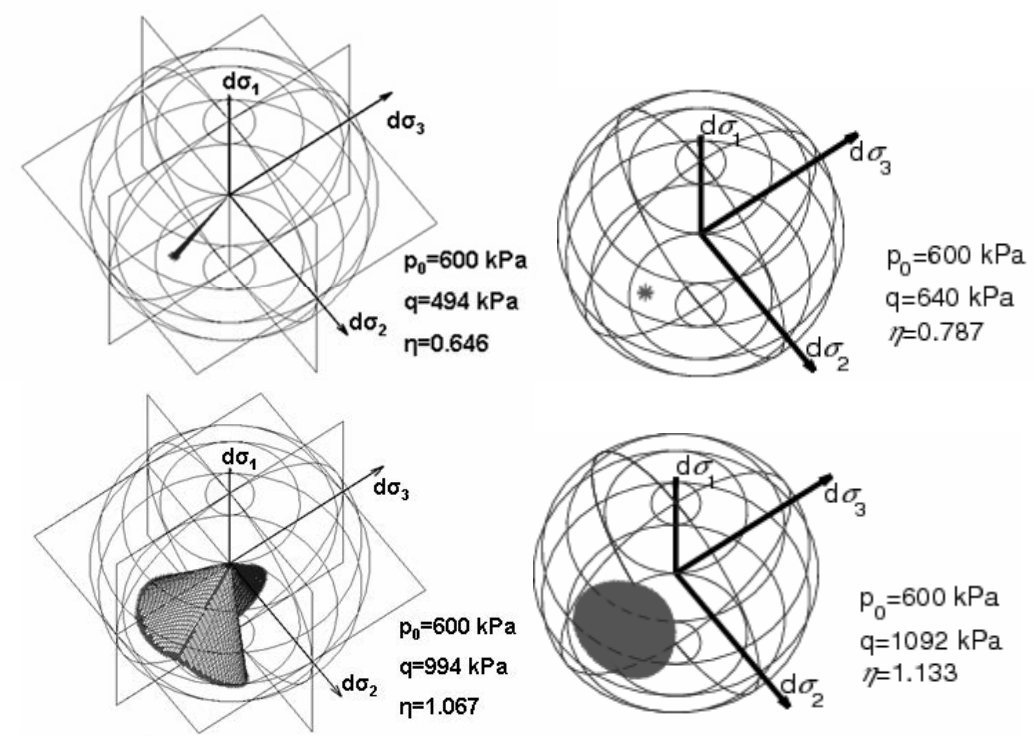

$\mathrm{p}_{0}=600 \mathrm{kPa}$ $\mathrm{q}=1092 \mathrm{kPa}$ $\eta=1.133$
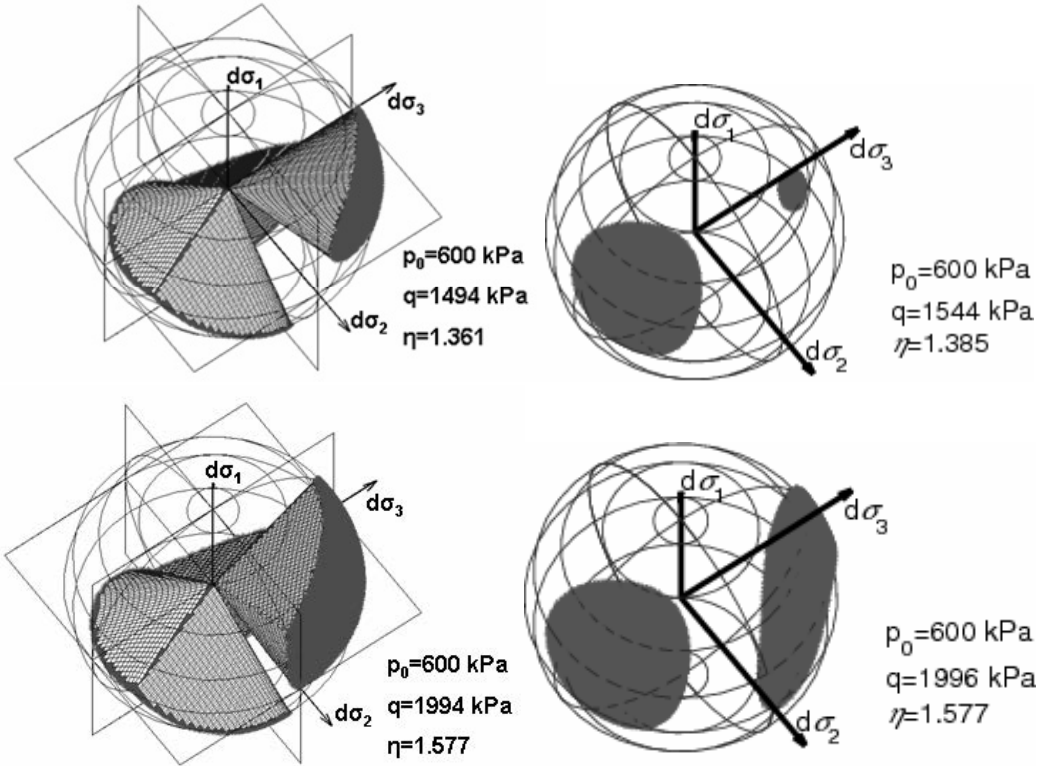

$p_{0}$ is the initial confining pressure, $q=\sigma_{1}-\sigma_{3}, \eta=\frac{q}{p}$

Figure 9.5. $3 D$ cones for dense Hostun sand, on the left: octo-linear model, on the right: non-linear model 


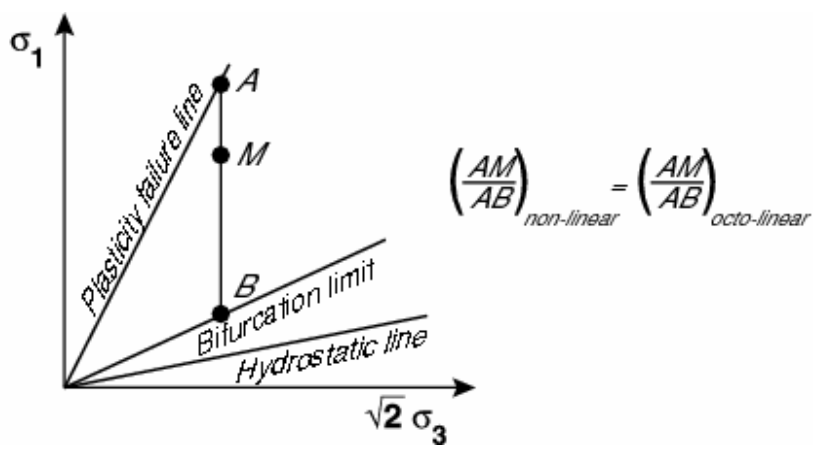

Figure 9.6. Mechanical points of comparison for the octo- and non-linear models

\subsection{Discrete modeling of the failure mode related to second-order work criterion}

Until now in this chapter, bifurcation analyses have been developed by considering a second-order work criterion applied to phenomenological constitutive relations. In this section a discrete 3D numerical model of granular materials is used to investigate 3 questions:

- does a granular material exhibit a bifurcation domain according to secondorder work criterion as shown previously?

- are there some cones of unstable stress directions as also shown previously?

- if the stress state is inside the bifurcation domain, the incremental stress direction inside the instability cone and if proper control parameters are considered, is an effective failure mode reached?

The utilized discrete element model, called SDEC, has been developed by [MAG 98] and, as is usual for molecular dynamic methods, each spherical grain interacts with its neighbors through basic local mechanical relations. The local elastic-plastic model is characterized by a normal elastic stiffness coefficient $k_{n}$, a tangential elastic stiffness coefficient $k_{t}$ and a local friction angle $\varphi_{c}$. In the present case, $\frac{k_{n}}{d_{s}}=356 \mathrm{MPa}$, with $d_{s}$ the sphere diameter, $\frac{k_{t}}{k_{n}}=0.42$ and $\varphi_{c}=35^{\circ}$. A schematic diagram of the grain-to-grain interaction is given in Figure 9.7b. A numerical cubical specimen of about 10,000 spheres can thus be built (Figure 9.7a) and some loading paths can be numerically applied to the boundary of the cube. 


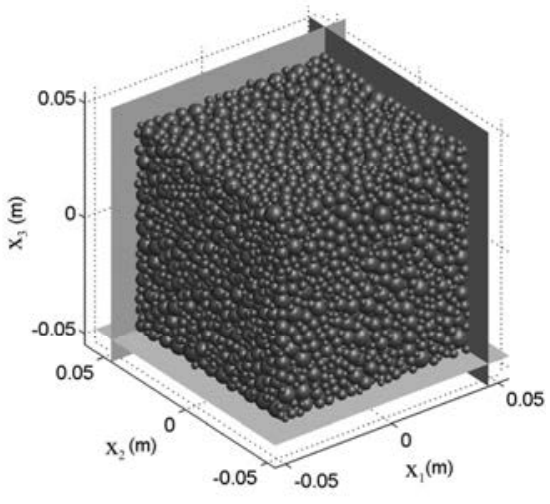

(a)

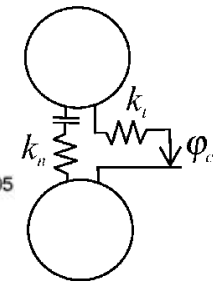

(b)

Figure 9.7. The discrete element model (a), rheological model of the inter-granular interaction (b)

This direct numerical simulation of the behavior of granular materials has revealed a remarkable capacity to realistically reproduce their "true" behavior, even if the mechanical ingredients are very rough, i.e. a simple elastic-plastic contact law at the meso-scale level. As generally for molecular dynamics, the macroscopic behavior extreme complexity is due to the very large number of interacting grains.

Thus the first objective was to investigate the polar variations of the secondorder work with respect to the incremental stress directions. Axisymmetric conditions are chosen, isotropic loadings are applied, then for 3 different pressure levels $\left(\sigma_{3}=100 ; 200 ; 300 \mathrm{kPa}\right)$ triaxial compressions are simulated and eventually at 4 different deviatoric stress levels $\left(\right.$ characterized by: $\left.\eta=\frac{q}{p}=\frac{\sigma_{1}-\sigma_{3}}{\frac{1}{3}\left(\sigma_{1}+2 \sigma_{3}\right)}\right)$ the values of second-order work are calculated in each stress directions from $10^{\circ}$ to $10^{\circ}$. The results are given in Figure 9.8 in polar diagrams versus the stress directions. Indeed normalized values of second-order work are considered in order to have values varying between -1 and +1 since it is the cosine of the angle between incremental stress and incremental strain:

$$
d^{2} W_{n o r m}=\frac{\underline{\underline{d \sigma}}: \underline{\underline{\underline{d \varepsilon}}}}{\|\underline{\underline{d \sigma}}\| \cdot\|\underline{\underline{d \varepsilon}}\|}
$$


Furthermore, a constant is added in order to see clearly in the figures when the values are negative. Thus the dashed circles on Figure 9.8 represent the zero values of second-order work, the points outside corresponding to positive values and inside to negative values (the principle of these diagrams was first presented in [DAR 00]). Figure 9.8 shows clearly that the second-order work can take negative values inside a cone of stress directions for high deviatoric stress levels and that this cone is oriented (roughly speaking) towards the stress space origin as was exhibited in section 9.2. All the results have been synthesized in Figure 9.9.

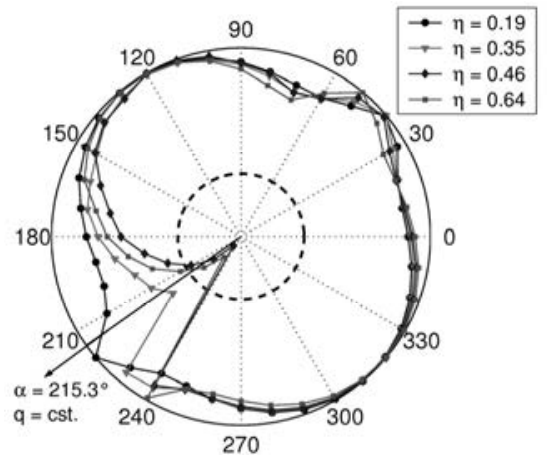

(a) $\sigma_{3}=100 \mathrm{kPa}$

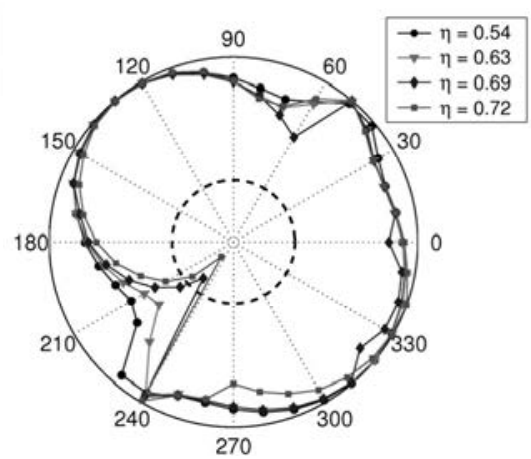

(b) $\sigma_{3}=200 \mathrm{kPa}$

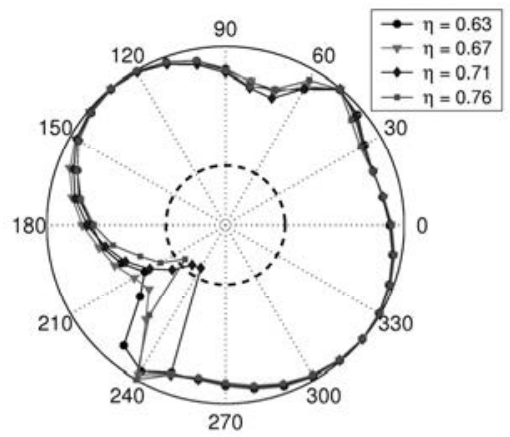

(c) $\sigma_{3}=300 \mathrm{kPa}$

Figure 9.8. Polar diagrams of the normalized second-order work $d^{2} W_{\text {norm }}$ calculated with a loose numerical specimen; dashed circles represent vanishing values of the second-order work

The so-called Rendulic plane for axisymmetric stress states is considered in Figure 9.9. All the stress points where the second-order work values have been 
checked are represented. For some of them, cones of negative values have been obtained and these cones are plotted. We see first in this figure that a bifurcation domain, which has an approximated conical shape whose apex would be at the plane origin, exists. Then secondly some instability cones appear for some stress states inside this bifurcation domain. All these cones are oriented towards the stress origin. Two specimens of different densities have been considered, a dense one (with a void ratio $e=0.618$ ) exhibiting a dilatant behavior during a triaxial drained compression, and a loose one ( $e=0.697)$ mainly contractant. The comparison between Figure $9.9 \mathrm{a}$ and Figure 9.9b shows the influence of the density on the bifurcation domain and the cones of unstable stress directions. The bifurcation domain is reduced for the denser specimen whereas it is more extended for the loose specimen. In the same way, the cones with the largest opening are obtained with the loosest specimen. Thus, the possibilities of bifurcation would seem more important for the loosest specimen. In addition, the directions included in the cones of unstable stress directions depend on the density. For instance, the direction corresponding to the constant stress deviator loading path $\left(d \sigma_{1}=d \sigma_{3}<0\right.$ such as $\left.d q=0\right)$ is included in cones for the loosest specimen (see Figure 9.8a) and not for the densest specimen. A qualitative identical influence of the density on the results is found with the Hostun sand [DAR 04].

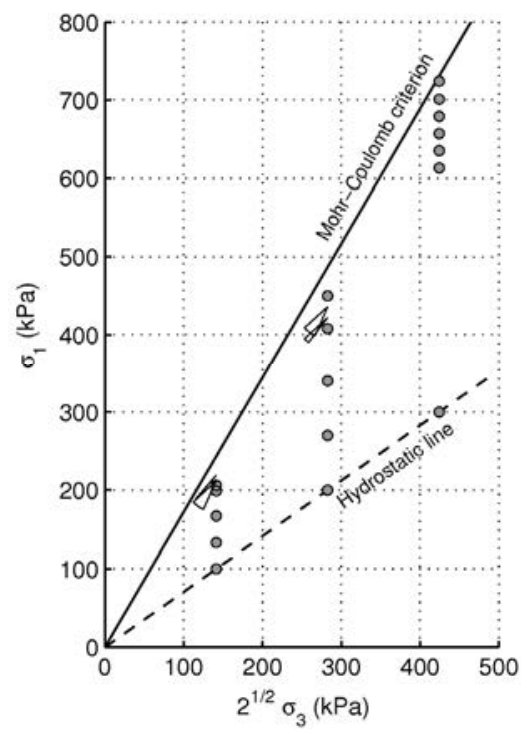

(a)

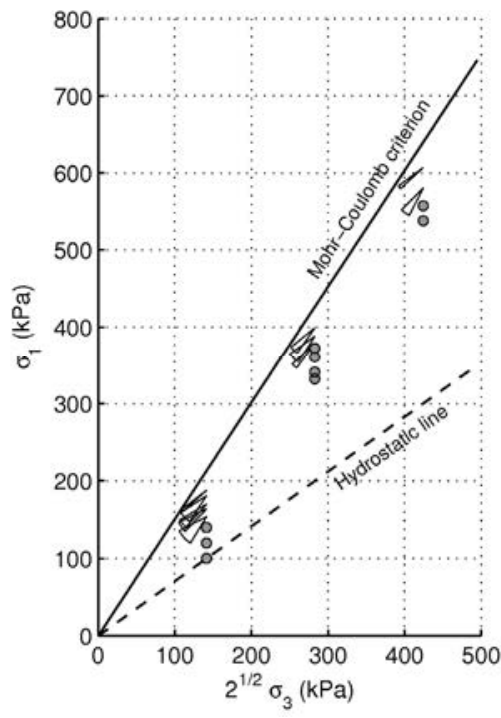

(b)

Figure 9.9. Synthesis of cones of unstable stress directions in the axisymmetric plane of stresses calculated from a dense numerical specimen (a) and a loose one (b); full circles represent stress probes for which no vanishing or negative values of $d^{2} W$ were found 
Until now it has been shown that the same type of bifurcation domain and instability cones can be obtained with phenomenological constitutive relations (section 9.2) and with direct numerical simulations by a discrete element method (this section). Another basic question is linked to the failure itself. If we take into account the 3 theoretical conditions for an effective failure:

$-\mathrm{C} 1$ : the stress state must be inside the bifurcation domain;

$-\mathrm{C} 2$ : the present stress direction must be inside the instability cone;

- C3: the loading parameters must be such that the failure is effective.

Is the discrete numerical model able to simulate a "true" failure, true failure being characterized by exponentially growing strains and a burst of kinetic energy? This question is discussed now.

Axisymmetric drained q cst loading paths have been studied experimentally and some failures have been obtained with loose sands before reaching the MohrCoulomb criterion [CHU 03]. This path is characterized by identical negative incremental stresses (q cst implies $\mathrm{d}_{1}=\mathrm{d} \sigma_{3}$ and the axisymmetric condition imposes $d \sigma_{2}=d \sigma_{3}$ ), thus it is in fact an incrementally isotropic unloading. In fact, for a loose sand we have established that this stress direction can be located inside the instability cone [DAR 04] and in fact Figure 9.8 shows that in case (a) (the initial isotropic pressure is equal to $100 \mathrm{kPa}$ ) for high deviatoric stress levels the $\mathrm{q}$ cst stress direction is inside the cone. Thus, conditions $\mathrm{C} 1$ and $\mathrm{C} 2$ are satisfied and it is interesting to compare the numerical responses of the cubical specimen when condition $\mathrm{C} 3$ is fulfilled or not. The theoretical analysis of these paths [DAR 04] shows that the proper loading or control parameters are $\mathrm{q}$ and $\varepsilon_{\mathrm{v}}$ (where $\varepsilon_{\mathrm{v}}$ is the first strain invariant or the opposite of the volume variation) for an effective failure. It means that, according to the theory, for control parameters defined by $\mathrm{d} \sigma_{1}=\mathrm{d \sigma}_{2}=$ $\mathrm{d \sigma}_{3}=$ given negative cst, the path should be stable until the Mohr-Coulomb criterion, while for control parameters defined by $\mathrm{dq}=0$ and $\mathrm{d} \varepsilon_{\mathrm{v}}=$ given negative cst, the path should be unstable.

Figure 9.10 shows the comparison between the results obtained with both sets of control parameters. Figure 9.10a presents the axial strain variation versus the computation time to reach the equilibrium of the sphere assembly. For a complete stress controlled path (by $\mathrm{d}_{1}, \mathrm{d \sigma}_{2}, \mathrm{d \sigma}_{3}$ ) the variation of the strain remains negligible while for a mixed control (by dq and $\mathrm{d} \varepsilon_{\mathrm{v}}$ ) the axial strain is growing exponentially (the lateral strain is also growing exponentially because of the link between both strains due to the imposed value of $d \varepsilon_{\mathrm{v}}$ ). Figure 9.10c gives the stress variations (the 3 principal stresses necessarily have the same variations). For a complete stress control, nothing specific appears, while for a mixed control the stresses decrease suddenly until 0 showing that the path is no more "controllable" in Nova's sense 
[NOV 94]. Finally, Figure 9.10a shows the variation of kinetic energy, calculated by adding the kinetic energy of each sphere in the cubical specimen. A burst of kinetic energy is clearly visible on Figure 9.10b for the mixed controlled loading path. In fact, it has been shown from theoretical considerations based on energy conservation that negative values of second-order work are linked to positive values of the increment of kinetic energy [NIC 07a]. In conclusion, a true failure mode is obtained numerically by a discrete element method in the precise theoretical conditions (see $\mathrm{C} 1, \mathrm{C} 2$ and $\mathrm{C} 3$ ) predicted by the theory developed in section 9.2.

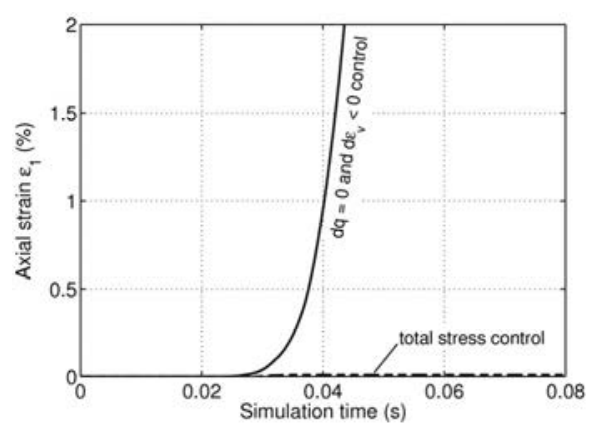

(a)

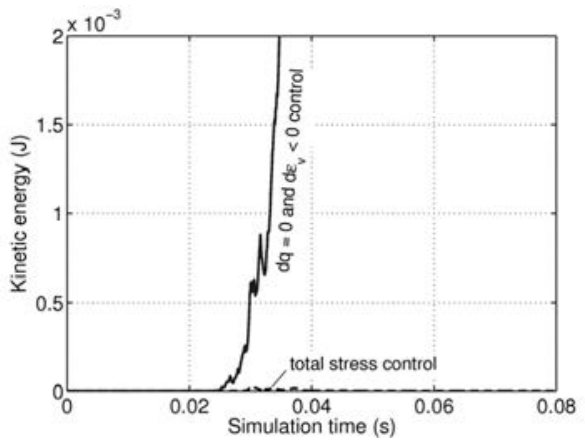

(b)

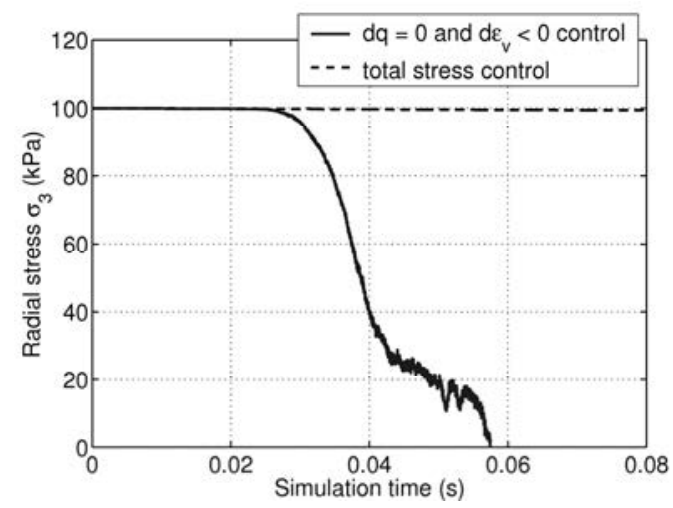

(c)

Figure 9.10. Comparison of the responses along a q-constant path simulated with a loose specimen for a full stress control and a control in $d q=0$ and dev $<0$

As previously, condition C3 (i.e. the question of the choice of the loading parameters) has been investigated, conditions $\mathrm{C} 1$ and $\mathrm{C} 2$ being fulfilled. Now we consider the case where $\mathrm{C} 1$ and $\mathrm{C} 3$ are satisfied and condition $\mathrm{C} 2$ is investigated. 
For that we have to choose a stress state inside the bifurcation domain, proper control parameters and to consider different loading directions, some of them inside the instability cone and others outside. The chosen loading paths are defined in axisymmetric conditions (they are in fact particular cases of the 3D paths considered in section 9.2) by:

$$
\left\{\begin{array}{c}
d \sigma_{1}-\frac{d \sigma_{3}}{R}=0 \\
d \varepsilon_{1}+2 R d \varepsilon_{3}=c s t \quad c s t \in \Re^{-} \quad(\text { here } c s t=-0.002 \%)
\end{array}\right.
$$

Let us note that the paths considered previously correspond to $\mathrm{R}=1$. For different values of $\mathrm{R}$, different stress directions can be obtained, they are characterized by various stress angles $\alpha$ as defined in Figure 9.8a (the initial isotropic pressure is equal to $100 \mathrm{kPa}$ ).

According to Figure 9.8a, the stress directions corresponding to $200^{\circ}(\mathrm{R}=1.94)$ and $240^{\circ}(\mathrm{R}=0.408)$ are outside the instability cone, $210^{\circ}(\mathrm{R}=1.22)$ and $230^{\circ}$ $(\mathrm{R}=0.593)$ are more or less close to the limits of the cone, and $220^{\circ}(\mathrm{R}=0.843)$ and $215.3^{\circ}(\mathrm{R}=1.00$, i.e direction corresponding to the $\mathrm{q}$ cst loading path considered previously) are inside the cone. The relation between stress angle $\alpha$ and $\mathrm{R}$ is given by:

$$
R=\frac{\operatorname{cotan}(\alpha)}{\sqrt{2}}
$$

The simulations consist of applying numerically to the same specimen at the same stress-strain state inside the bifurcation domain (i.e. the state considered on Figure 9.8a with $\eta=0.46$ ) the 6 incremental loadings oriented towards the 6 stress directions defined above.

The results are given in Figure 9.11. For the stress angles equal to $200^{\circ}$ or $240^{\circ}$, nothing happens. The kinetic energy of the specimen remains equal to zero, and the stresses and the strains do not vary significantly. For this stress-strain state located inside the bifurcation domain and thus potentially unstable, an infinitesimal loading is applied in a stable direction outside the instability cone. According to the theory developed in section 9.2 no failure is expected.

Let us consider now the stress angles $210^{\circ}$ and $230^{\circ}$. A small variation of the kinetic energy is visible in both cases in Figure 9.11, but rapidly the kinetic energy vanishes again. Some limited variations of lateral strain and lateral stress (and thus of the axial strain and stress) are induced. For $\alpha=210^{\circ}$ a new stress-strain 
equilibrium state is reached which differs in a random way to the initial state whereas, for $\alpha=230^{\circ}$, the specimen totally collapses suddenly. These stress directions are close to the limits of the instability cone and the response to a small loading in these directions depends on imperfections, of a numerical nature in these calculations or of a mechanical nature in experimental tests. The stress-strain response is finite and random. We know that such a bifurcated response is not deterministic.

The last two stress directions of $215.3^{\circ}$ and $220^{\circ}$ are neatly inside the instability cone. In both cases, a large burst of kinetic energy is visible in Figure 9.11, the strains are unlimited and the stresses vanish. The specimen has liquefied, if the usual definition of liquefaction is accepted, i.e. vanishing of inter-granular stresses as a mechanical response of the material to a strain controlled or mixed controlled loading path [DAR 96]. A true failure state has been reached, as expected from the present theory.
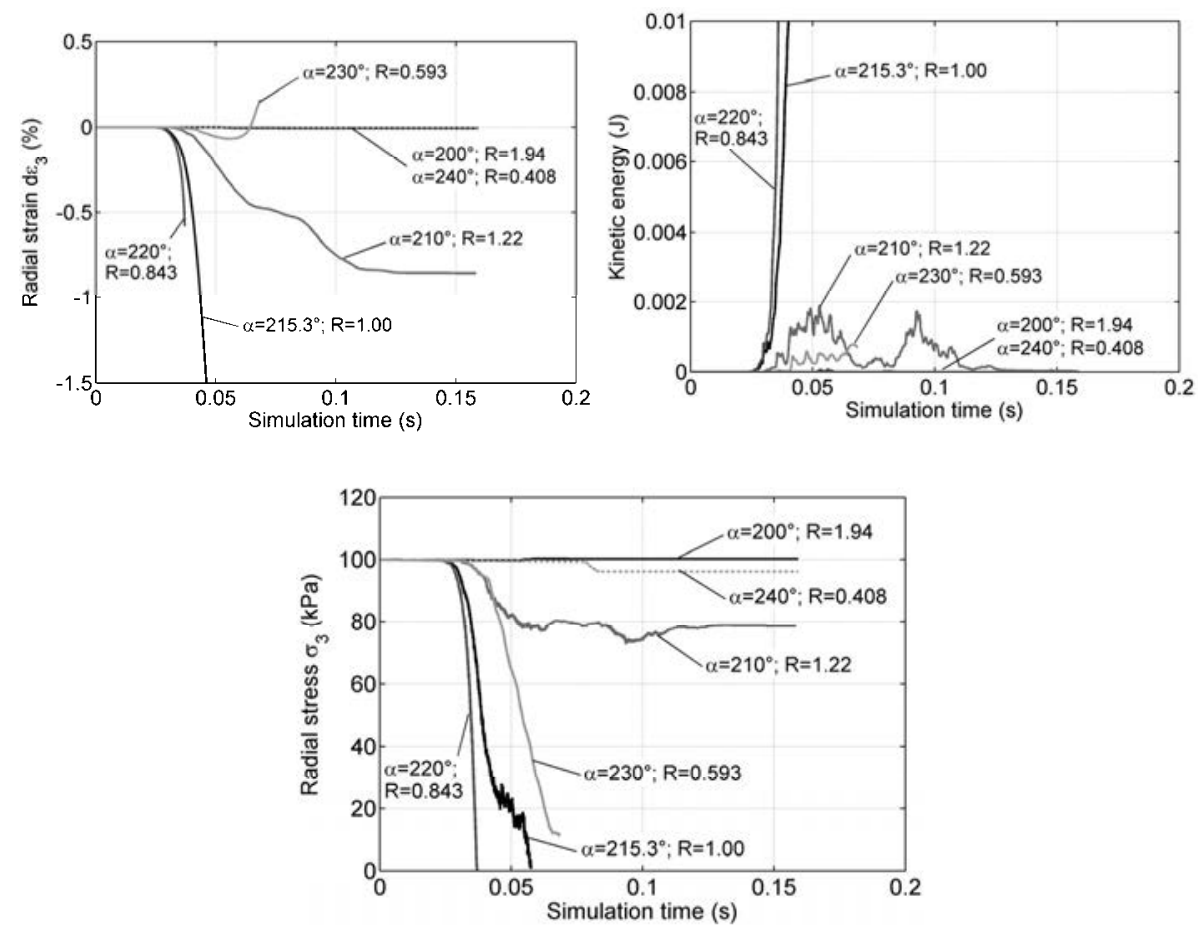

Figure 9.11. Simulated responses with the loose specimen to the application of the loading program $d s 1-d s 3 / R=0$ and de $1+2 R$ de $3<0$ from the stress state $d s 3=100 \mathrm{kPa}$ and eta $=0.46$ (different $R$ values are considered) 
It is interesting to note that this failure mode is a "diffuse" one, and not a "localized" one, if we call "diffuse" a failure mode without any kind of strain localization by shear band, compaction band or dilation band [DAR 04]. In fact, in each of these numerical simulations only chaotic and random displacement fields without any localization figure were observed at failure, which is also confirmed by the strain localization theory since the localization criterion (vanishing values of the determinant of the acoustic tensor) is not satisfied for these stress states. In fact, when the determinant of the acoustic tensor is zero, the determinant of the symmetric part of the elastoplastic tensor necessarily vanishes, which means that the second-order work criterion is satisfied before the localization criterion (but of course not necessarily in the same stress directions).

\subsection{Conclusions}

In the first part of this chapter, a 3D analysis of second-order work criterion was proposed.

This criterion which is essentially a material instability criterion (some small perturbations of the loading or of the mechanical state induce large responses) is associated with bifurcation points (the mechanical state is changing suddenly with a burst of kinetic energy). The general equation of the boundary of the bifurcation domain has been given for elastoplastic incrementally piecewise linear constitutive relations. The corresponding surface in the 3D principal stress space is approximately a cone whose apex is at the space origin for a non-cohesive material [PRU 08]. The approximately conical structure of the Mohr-Coulomb plastic criterion is thus preserved for the bifurcation limit surface. If we except flutter instabilities, all possible failure modes are inside this bifurcation domain.

Due to the directional structure of second-order work criterion, there are unstable stress directions and the existence of some instability cones has been analytically proved and numerically verified. It appears that there is one cone (at the maximum) in a given elastoplastic tensorial zone. The $3 \mathrm{D}$ equations of the instability cones have been established. In a given tensorial zone, these cones have been shown to be elliptical, which implies that in a general manner the unstable stress directions form sets of elliptical cones [PRU 08].

In classical plastic theory, two relations have to be fulfilled: the plastic limit criterion and the flow rule. In the same way, it has been shown here that in addition to the bifurcation criterion a failure rule has to be satisfied. This failure rule is clearly distinguishable from a flow rule, because it is a mixed incremental stressstrain relation. It shows that, even if the solution is no longer unique as along a flow 
rule, the infinite number of solutions has to satisfy some common conditions given by this failure rule.

In a second part, these analyses have been performed with a discrete element method on numerical cubical specimens [SIB 07a]. The same main features have been obtained: existences of a bifurcation domain whose limit is approximately a straight line passing through the stress plane origin for axisymmetric conditions, and of instability cones directed towards the origin. Then the 3 theoretical conditions to obtain an effective failure have been examined:

$-\mathrm{C} 1$ : the stress state must be inside the bifurcation domain;

- C2: the stress direction must be inside the instability cone;

- C3: the loading variables must correspond to the bifurcation criterion.

Knowing that $\mathrm{C} 1$ is obvious, the numerical simulations have shown that, if $\mathrm{C} 2$ or $\mathrm{C} 3$ are not satisfied, no failure appears, while if $\mathrm{C} 2$ and $\mathrm{C} 3$ are both fulfilled a true failure mode suddenly develops. This failure is characterized by exponentially growing strains, by vanishing stresses and by bursts of kinetic energy [SIB 07b]. No localization figure is observed, which is a good indicator of a diffuse failure mode.

\subsection{Acknowledgements}

These theoretical and numerical results have been used to assess the instability of landslides and rock falls, thanks to the support of 2 European PCRD projects (DIGA and LESSLOSS) and 3 national French ANR projects (ROMICO, SIGMA and STABROCK). Without this support probably none of this research would have been developed.

\subsection{Bibliography}

[BIG 90] Bigoni D., Hueckel T., Uniqueness and localization I. Associative and nonassociative elastoplasticity, Int. J. Solids Structures, 28(2), 197-213, 1991.

[CHU 03] Chu J., LeONG W., Recent progress in experimental studies on instability of granular soil, LABUZ and DRESCHER (Ed.), Int. Workshop on Bifurcations and Instabilities in Geomechanics, Swets \& Zeitlinger, 175-191, 2003.

[DAR 95] Darve F., Flavigny E., Meghachou M., Yield surfaces and principle of superposition revisited by incrementally non-linear constitutive relations, International Journal of Plasticity, 11(8), 927-948, 1995.

[DAR 96] DARVE F., Liquefaction phenomenon of granular materials and constitutive stability, Int. J. Eng. Computations, 13(7), 5-28, 1996. 
[DAR 00] DARVE F., LAOUAFA F., Instabilities in granular materials and application to landslides, Mechanics of cohesive-frictional materials, 5, 627-652, 2000.

[DAR 04] Darve F., Servant G., Laouafa F., Khoa H.D.V., Failure in geomaterials: continuous and discrete analyses, Comput. Meth. Appl. Mech. Eng., 193(27-29), 30573085, 2004.

[DER 04] Desrues J., Viggiani G., Strain localization in sand: an overview of the experimental results obtained in Grenoble using stereophotogrammetry, Int. Journal for Numerical and Analytical methods in Geomechanics, 28(4), 279-321, 2004.

[GUD 79] GUDEHUS G., A comparison of some constitutive laws for soils under radially loading symmetric loading and unloading, in 3rd International Conference on Numerical Methods in Geomechanics, A. Balkema (2), 1309-1323, 1979.

[HIL 58] HILl R., A general theory of uniqueness and stability in elasto-plastic solids, Journal of the Mechanics and Physics of Solids, 6, 236-249, 1958.

[KHO 06] Khoa H.D.V., Georgopoulos I.O., Darve F., Laouafa F., Diffuse failure in geomaterials: experiments and modelling, Computer and Geotechnics, 33, 1-14, 2006.

[MAG 98] MAGNIER S.A., DonZÉ F.V., Numerical simulations of impacts using a discrete element method, Mech. Cohes. Frict. Mater., 3, 257-276, 1998.

[NIC 07a] NicOt F., DARVE F., A micro-mechanical investigation of bifurcation in granular materials, International Journal of Solids and Structures, 44(20), 6630-6652, 2007.

[NIC 07b] Nicot F., Darve F., KhoA H.D.V., Bifurcation and second-order work in geomaterials, Int. J. Num. Anal. Methods in Geomechanics, in press, 2007.

[NOV 04] Nova R., Controllability of the incremental response of soil specimens subjected to arbitrary loading programmes, J. Mech. Behav. Mater., 5(2), 193-201, 2004.

[PRU 08] Prunier F, Nicot F, Darve F, Laouafa F, Lignon S, 3D Multi scale bifurcation analysis of granular media, submitted.

[SIB 07a] Sibille L., Nicot F., Donzé F.V., Darve F., Material instability in granular assemblies from fundamentally different models, Int. J. Numer. Anal. Meth. Geomech., 31, 457-481, 2007.

[SIB 07b] Sibille L., Donzé F.V., Nicot F., Chareyre B., Darve F., From bifurcation to failure in a granular material, a DEM analysis, Acta Geotechnica, online, 2007. 